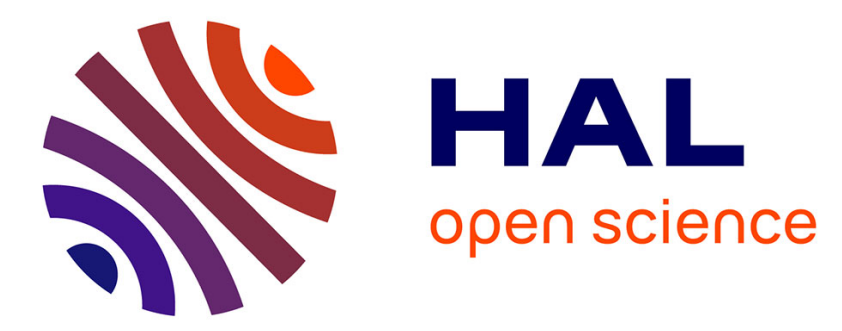

\title{
QUELQUES ASPECTS FONDAMENTAUX DANS LE FONCTIONNEMENT DES CHAMBRES A MIGRATION
}

J. Gresser, G. Schultz

\section{- To cite this version:}

J. Gresser, G. Schultz. QUELQUES ASPECTS FONDAMENTAUX DANS LE FONCTIONNEMENT DES CHAMBRES A MIGRATION. Congres de la Societe Francaise de Physique, 1977, Poitiers, France. pp.C3-22-C3-32, 10.1051/jphyscol:1978305 . jpa-00217409

\section{HAL Id: jpa-00217409 \\ https://hal.science/jpa-00217409}

Submitted on 1 Jan 1978

HAL is a multi-disciplinary open access archive for the deposit and dissemination of scientific research documents, whether they are published or not. The documents may come from teaching and research institutions in France or abroad, or from public or private research centers.
L'archive ouverte pluridisciplinaire HAL, est destinée au dépôt et à la diffusion de documents scientifiques de niveau recherche, publiés ou non, émanant des établissements d'enseignement et de recherche français ou étrangers, des laboratoires publics ou privés. 


\title{
QUELQUES ASPECTS FONDAMENTAUX DANS LE FONCTIONNEMENT DES CHAMBRES A MIGRATION
}

\author{
J. GRESSER \\ Laboratoire d'Électronique et d'Instrumentation Nucléaire, Université du Haut-Rhin, Mulhouse, France \\ et G. SCHULTZ (*) \\ Centre de Recherches Nucléaires (Laboratoire PNPP-HE), Université Louis-Pasteur, 67037 Strasbourg, \\ France
}

\begin{abstract}
Résumé. - En vue d'optimiser le fonctionnement des chambres à migration, différents coefficients de transport (vitesse, coefficient de diffusion, énergie caractéristique) de l'électron ont été calculés pour différentes valeurs de champ électrique et pour différents gaz (argon - gaz carbonique - isobutane - méthane - méthylal). Le comportement de l'électron dans les mélanges gazeux argon-isobutane est étudié particulièrement ainsi que l'action d'une faible quantité de méthylal sur ce comportement. L'influence de variations de pression et de température sur la vitesse de migration est également analysée. Quelques remarques intéressantes sont formulées à propos de la précision spatiale de détection des chambres à migration et de la limite intrinsèque de cette précision.
\end{abstract}

\begin{abstract}
Different transport coefficients (velocity, diffusion coefficient, characteristic energy) of electrons have been calculated for different electric field values and different gases (argon, carbon dioxide, isobutane, méthane, méthylal) in order to find the optimum operating conditions for drift chambers. We have also studied the behaviour of electrons in argon-isobutane mixtures and the influence of a small addition of methylal on this behaviour. The influence of variations of pressure and temperature on drift velocity is also studied. Some conclusions are also given about the spatial precision of detection in drift chambers and the intrinsic limit of this precision.
\end{abstract}

L'avènement d'accélérateurs de plus en plus puissants a accentué le développement des chambres à migration ces dernières années. Ce développement s'est fait essentiellement à partir des connaissances acquises sur les chambres proportionnelles et à l'aide de résultats expérimentaux décrivant le comportement de l'électron de migration dans différents gaz ou mélanges gazeux en fonction de la valeur du champ électrique.

Cependant, V. Palladino et B. Sadoulet ont publié deux articles de synthèse $[1,2]$ dans lesquels ils exposent les résultats de différents calculs décrivant le comportement des électrons dans certains gaz. Nous nous sommes inspirés de ce travail pour étudier la migration des électrons en fonction du champ électrique pour cinq gaz utilisés couramment dans les chambres proportionnelles et dont les propriétés d'amplification sont assez bien connues (argon, isobutane, gaz carbonique, méthane, méthylal) $[3,4]$.

Le calcul qui prend en compte des relations simplifiées permet d'évaluer différents paramètres caractérisant le comportement des électrons dans un gaz ou mélange gazeux, à savoir :

(*) Adresse actuelle : Laboratoire d'Électronique et d'Instrumentation Nucléaire, Université du Haut-Rhin, Mulhouse, France.
- la vitesse de migration de l'électron,

- le coefficient de diffusion de l'électron,

- l'énergie caractéristique de l'électron, elle-même liée à la fluctuation statistique de la position de l'électron à un temps donné.

Nous résumons dans cet article certains résultats intéressants qui permettent d'optimiser le fonctionnement des chambres à migration.

Nous présentons d'abord et brièvement certains aspects théoriques de la migration des électrons dans les gaz. Nous étudierons ensuite la migration des électrons dans quelques gaz ou mélanges gazeux où le comportement des électrons est relativement stable (faible influence des variations de pression $\Delta p$ et de température $\Delta T$ ) avant de justifier le choix du mélange gazeux standard utilisé au C.E.R.N. (groupe Charpak-Sauli) (argon $66,4 \%$, isobutane $30,2 \%$ et méthylal $3,4 \%$ ) ( $\left.{ }^{1}\right)$. Nous analyserons ensuite la dispersion spatiale des électrons pour le

(1) Dans différentes publications [5, 6], le mélange standard utilisé au C.E.R.N. a été présenté comme étant constitué de $67,2 \%$ d'argon, $30,3 \%$ d'isobutane et $2,5 \%$ de méthylal. Certaines incompatibilités entre les résultats de nos mesures et les valeurs théoriques données par notre calcul nous ont amené à recalibrer le banc de mélange de gaz. Les nouvelles proportions de gaz permettent de trouver un bon accord entre valeurs théoriques et expérimentales, notamment pour la variation de la vitesse de migration $w_{x}$ en fonction du champ électrique. 
mélange standard et les conséquences sur la précision spatiale de détection des chambres à migration.

1. Aspect théorique. - C'est en 1935 que P. M. Morse, W. P. Allis et E. S. Lamar publient un article fondamental [7] décrivant le comportement des électrons libres dans un gaz sous l'influence d'un champ électrique. L'énergie cinétique de l'électron est supposée être plus grande que l'énergie thermique des atomes ou molécules de gaz, mais cependant assez faible pour que le choc avec les atomes ou molécules de gaz reste élastique. Leur calcul aboutit à la fonction de distribution de l'énergie des électrons suivante :

$$
F_{0}(\mathscr{E})=C \sqrt{\mathscr{E}} \exp -\int \frac{3 \Lambda \mathscr{E} \mathrm{d} \mathscr{E}}{\left[e E l_{c}(\mathscr{E})\right]^{2}}
$$

où $\Lambda=2 \mathrm{~m} / M$ est la fraction moyenne d'énergie perdue par l'électron lors d'un choc, $l_{e}(\mathscr{E})$ le libre parcours moyen de l'électron entre deux chocs et $C$ une constante de normalisation $\left(F_{0}(\mathscr{E}) \mathrm{d} \mathscr{E}=1\right)$.

Si l'énergie des électrons qui migrent est de l'ordre de grandeur de l'énergie thermique des atomes ou molécules de gaz $(\mathscr{E} \approx K T \approx 0,025 \mathrm{eV}$ à température ambiante), il faut tenir compte du mouvement des molécules [8] ce qui conduit à la fonction de distribution suivante :

$$
F_{0}(\mathscr{E})=C \sqrt{\mathscr{E}} \exp -\int \frac{3 \Lambda \mathscr{E} \mathrm{d} \mathscr{E}}{\left[e E l_{\mathrm{e}}(\mathscr{E})\right]^{2}+3 \Lambda \mathscr{E} K T} .
$$

Il est à remarquer que si le terme $\left(e E l_{\mathrm{e}}\right)^{2}$ est faible devant $3 \Lambda^{\mathscr{E}} K T$, la fonction de distribution est celle, bien connue, de Maxwell :

$$
F_{0}(\mathscr{E})=C \sqrt{\mathscr{E}} \exp -\frac{\mathscr{E}}{K T} .
$$

L'équation (2) convient parfaitement pour l'étude du comportement des électrons dans l'argon pour des champs électriques inférieurs à $1000 \mathrm{~V} / \mathrm{cm}$ (voir paragraphe 3.1). Cependant, pour les autres gaz qui nous intéressent (gaz carbonique, isobutane, méthane et méthylal), des chocs d'excitation du type rotationnel ou vibrationnel se produisent à des énergies relativement faibles (quelques dixièmes d'eV). Dans ce cas, la relation (2) convient à condition que $\Lambda$ soit remplacé par:

$$
\Lambda(\mathscr{E})=\frac{2 m}{M}+\sum_{h} \frac{\mathscr{E}_{h}}{\mathscr{E}} \frac{l_{\mathrm{e}}(\mathscr{E})}{l_{h}(\mathscr{E})}
$$

où $\mathscr{E}_{h}$ est l'énergie d'excitation du $h$-ième niveau excité et $l_{h}$ le libre parcours moyen entre deux collisions donnant naissance à l'excitation des électrons du niveau $h$.

Cette dernière relation peut s'écrire autrement en introduisant :

- le nombre de Loschmidt

$$
N=N_{\mathrm{o}} \times \frac{p}{760} \times \frac{273}{T}
$$

avec $N_{0}=$ nombre de Loschmidt défini à $0^{\circ} \mathrm{C}$ et à pression atmosphérique $=2,6871 \times 10^{25}$ molécules $\times \mathrm{m}^{-3}$

$p=$ pression du gaz en torr

$T=$ température absolue du gaz

- la section efficace

$$
\sigma(\mathscr{E})=\frac{1}{N l(\mathscr{E})}
$$

Dans ce cas :

$$
\Lambda(\mathscr{E})=\frac{2 m}{M}+\sum_{h} \frac{\mathscr{E}}{\mathscr{E}} \frac{\sigma_{h}(\mathscr{E})}{\sigma_{\mathrm{e}}(\mathscr{E})}
$$

En l'absence de champ magnétique, les coefficients de transport qui nous intéressent sont $[1,4]$ :

a) la composante de la vitesse de l'électron parallèle au champ électrique $E$

$$
w_{x}=-\frac{2}{3} \frac{e E}{m} \int \mathscr{E} l_{e}(\mathscr{E}) \frac{\partial F_{0}(\mathscr{E}) / v}{\partial \mathscr{E}} \mathrm{d} \mathscr{E}
$$

b) le coefficient de diffusion:

$$
D=\int \frac{l_{\mathrm{e}}(\mathscr{E})}{3} v F_{0}(\mathscr{E}) \mathrm{d} \mathscr{E}
$$

c) l'énergie caractéristique :

$$
\mathscr{E}_{k}=\frac{e D}{\mu}=\frac{e D E}{w_{x}}
$$

où $\mu$ est le coefficient de mobilité de l'électron dans le gaz. (Par définition: $w_{x}=\mu E$.)

d) la dispersion spatiale $\sigma_{x}$ des électrons.

$\mathrm{Si}$ on considère le cas simple où des électrons migrent pendant un temps $t$ dans un champ électrique constant $E$, la dispersion au point $x$ est :

$$
\sigma_{x}=\sqrt{2 D t}=\sqrt{\frac{2 \mathscr{E}_{k} x}{e E}}
$$

2. Mëthode de calcul. - Les relations exposées précédemment nous ont permis de préparer un programme de calcul qui permet d'établir les différents coefficients de transport de l'électron dans un gaz ou un mélange gazeux.

\subsection{RÉSUMÉ DES HYPOTHËSES SIMPLIFICATRICES.} Résumons les différentes hypothèses simplificatrices qui sont faites dans ce calcul :

- nous ne tenons pas compte des phénomènes d'ionisation effectivement négligeables pour les gaz considérés et aux énergies envisagées $(<10 \mathrm{eV})$,

- les phénomènes d'excitation électronique sont négligés. En effet, ceux-ci ne commencent que pour des énergies relativement élevées ( $4 \mathrm{eV}$ pour le gaz carbonique, 11,5 eV pour l'argon). Naturellement, il faut être prudent et vérifier que la fonction de distribution $F_{0}(\mathscr{E})$ est bien nulle aux énergies d'excitation électronique. 
Par contre, les phénomènes d'excitation vibrationnelle et rotationnelle, importants aux basses énergies doivent être pris en compte. Ils l'ont été par l'utilisation de la relation (6).

- La distribution des électrons est considérée comme uniforme donc indépendante de $x$. Ceci n'est pas vrai dans notre cas car les électrons migrent en paquets et engendrent de nouveaux courants de diffusion dus à la non-uniformité de la distribution. Mais ces courants sont généralement négligeables, comme l'ont montré J. H. Parker et J. J. Lowke [9] qui ont développé des calculs où $\partial F_{0} / \partial x$ n'était pas nul.

2.2 DETERMINATION DES GRANDEURS $\sigma_{\mathrm{e}}$ ET $\boldsymbol{\Lambda}$. Il faut introduire dans le programme de calcul deux grandeurs caractérisant le gaz, à savoir :

- la section efficace de collisions élastiques $\sigma_{\mathrm{e}}=\sigma_{\mathrm{e}}(\mathscr{E})$ liée à $l_{\mathrm{e}}(\mathscr{E})$ par les relations (4) et (5);

- la fraction moyenne d'énergie perdue par l'électron lors d'une collision $\Lambda=\Lambda(\mathscr{E})$ établie à l'aide de la relation (6) après avoir déterminé l'énergie d'excitation $\mathscr{E}_{h}$ du niveau $h$ et la section efficace $\sigma_{h}$ des collisions du type excitation concernant ce niveau.

Le calcul est étendu à des mélanges gazeux à l'aide des relations :

$$
\begin{gathered}
\sigma_{\mathrm{e}}=\sum_{i} \delta_{i} \sigma_{i} \\
\sigma_{e} \Lambda=\sum_{i} \delta_{i} \sigma_{i} \Lambda_{i}
\end{gathered}
$$

où $\delta_{i}$ est le pourcentage du $i$ ème gaz du mélange $\left(\sum_{i} \delta_{i}=1\right)$

Pour l'argon, ces deux grandeurs sont bien établies, ce qui nous a permis de tester le bon fonctionnement et la validité du programme. Pour le gaz carbonique, elles sont également connues mais la complexité des sections efficaces vibrationnelles nous a améné à des simplifications dont il sera parlé au paragraphe 3.2. Malheureusement, pour l'isobutane, le méthane et le méthylal, $\sigma_{\mathrm{e}}$ et $\Lambda$ sont mal connus et souvent sur une gamme restreinte d'énergie. Nous avons donc été obligés de déduire $\sigma_{\mathrm{e}}$ et $\Lambda$ à partir de mesures de la vitesse de migration $w_{x}$ en fonction du champ électrique $E$, mesures réalisées sur des gaz purs ou des mélanges.

Certaines mesures expérimentales sont publiées dans la littérature; d'autres ont été réalisées au C.E.R.N. dans le groupe dirigé par G. Charpak et F. Sauli à l'aide d'une chambre où un champ électrique extrêmement uniforme peut être appliqué [5].

3. Vitesse de migration dans différents gaz ou mélanges gazeux. - La relation espace-temps caractérisée par la vitesse de migration des électrons dépend du mélange gazeux choisi et du champ électrique qui règne dans la chambre. Ce champ n'est pas uniforme dans l'espace de migration et sa valeur passe par un minimum $E_{\mathrm{m}}$ voisin de $1,35 \mathrm{kV} / \mathrm{cm}$ dans notre cas $[3,5]$. Nous nous sommes attachés à trouver un mélange gazeux où la vitesse de migration soit constante et saturée pour des champs électriques supérieurs ou égaux à $E_{\mathrm{m}}$. La raison fondamentale qui nous a guidé dans ce choix est que si la vitesse de migration est constante quel que soit $E>E_{\mathrm{m}}$, celle-ci n'est pratiquement pas influencée par les variations de pression et de température. En effet, si on considère les expressions de la fonction de distribution $F_{0}$ [relation (2)] et de la vitesse de migration $w_{x}$ [relation (7)], on constate que le champ électrique $E$ apparaît sous la forme du produit $E l_{\mathrm{c}}$. Or, d'après les relations (4) et (5), $l_{c}$ varie comme l'inverse du nombre de Loschmidt $N$ donc comme l'inverse de la pression $p$. Aussi toute évolution de $w_{x}$ en fonction du champ électrique peut invariablement être présentée en fonction de $E / N$ ou $E / p$. $w_{x}$ ne varie donc pas avec $p$ si $w_{x}$ est constant quel que soit $E$.

Si l'énergie des électrons qui migrent est grande de sorte que l'on peut négliger l'effet du mouvement des atomes ou molécules de gaz sur la vitesse de migration des électrons, la fonction de distribution des énergies est celle de la relation (1). Dans l'expression de cette fonction de distribution et celle de la vitesse $w_{x}$, la température $T$ est alors liée à $E$ sous forme du produit $E \times T$ (d'après les relations (4) et (5)). Aussi la vitesse de migration ne varie-t-elle pas avec $T$ si $w_{x}$ reste constant en fonction de $E$.

Cependant, pour les mélanges qui nous intéressent, l'énergie des électrons de migration ne peut pas toujours être négligée par rapport à l'énergie thermique; dans ce cas, l'influence de la variation de température sur la vitesse de migration ne se réduit plus à une relation simple et $w_{x}$ peut varier avec $T$ même si pour une température donnée $w_{x}$ est constant en fonction de $E$. Mais cette variation est généralement faible aux champs électriques qui nous intéressent (supérieurs à $1,35 \mathrm{kV}$ ) et à pression et température normales.

Nous allons d'abord considérer le cas de l'argon pur, puis nous allons déterminer successivement le mélange argon-gaz carbonique, argon-méthane et argon-isobutane qui nous donne une vitesse de migration de l'électron constante à partir d'une certaine valeur de $\boldsymbol{E}$. Enfin nous allons analyser l'influence d'une faible quantité de méthylal sur la vitesse de migration des électrons dans les mélanges argon-isobutane.

3.1 ARGON. - La variation de la section efficace $\sigma_{\mathrm{e}}$ en fonction de l'énergie des électrons utilisée dans le calcul est celle employée par A. G. Engherardt et A. V. Phelps [10] d'une part et V. Palladino et B. Sadoulet $[1,2]$ d'autre part. Elle est représentée sur la figure 1. La paramétrisation de cette courbe, 


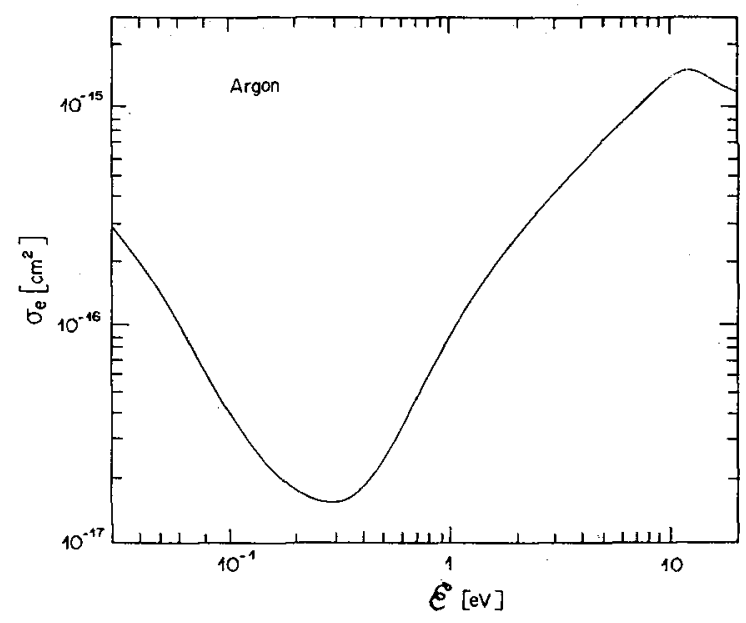

FIG. 1. - Section efficace de collisions élastiques des électrons dans l'argon. A noter le creux de Ramsauer à $0,3 \mathrm{eV}$.

nécessaire dans notre calcul, est détaillée en annexe. Sur la figure 2 nous montrons la vitesse de migration $w_{x}$ et l'énergie caractéristique $\mathscr{E}_{k}$ calculées en fonction de $E / N$ [voir relation (4)]. On constate que ces courbes sont en parfait accord avec les points relevés expérimentalement par différents auteurs et résumés par A. G. Engherardt et A. V. Phelps [10] et J. L. Pack, R. E. Voshall et A. V. Phelps [11] sauf pour $E / N>4 \times 10^{-17} \mathrm{~V} \times \mathrm{cm}^{2}$ (ce qui correspond à un champ électrique d'environ $1000 \mathrm{~V} / \mathrm{cm}$ à pression atmosphérique et à température ambiante). Dans ce cas, il apparaît des excitations électroniques que notre calcul n'envisage pas.

Il est à noter que le fait d'ajouter de l'isobutane, du gaz carbonique, du méthane ou du méthylal à

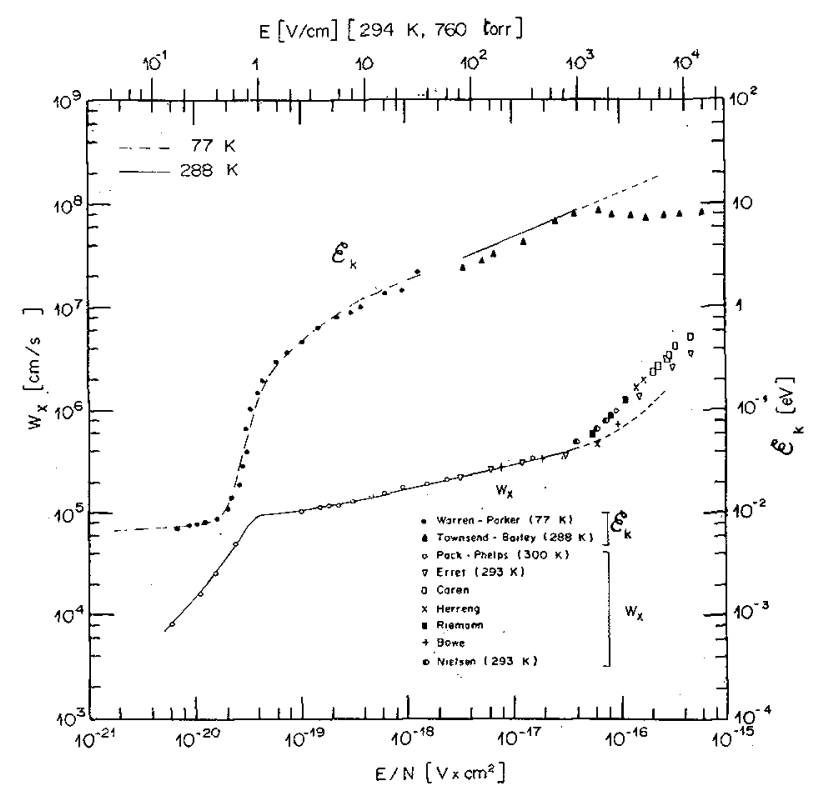

FIG. 2. - Vitesse de migration $w_{x}$ et énergie caractéristique $\mathscr{E}_{k}$ dans l'argon en fonction de $E / N$. Les courbes théoriques sont en parfait accord avec les points relevés expérimentalement par différents auteurs $[10,11]$ sauf pour $E / N>4 \times 10^{-17} \mathrm{~V} \times \mathrm{cm}^{2}$ (voir texte). l'argon diminue l'énergie moyenne des électrons et de ce fait notre modèle reste valable pour les mélanges gazeux qui nous intéressent et pour des champs électriques bien supérieurs à $1000 \mathrm{~V} / \mathrm{cm}$ (pression et température normales).

3.2 ARGON ET MÉLANGES Ar- $\mathrm{CO}_{2}$. - Une section efficace $\sigma_{\mathrm{e}}$ du gaz carbonique en fonction de l'énergie des électrons a été établie par R. D. Hake Jr, et A. V. Phelps [12]. Comme le soulignent ces auteurs, cette section efficace diffère légèrement de la section efficace totale $\sigma\left({ }^{2}\right)$ établie à partir de mesures avec des électrons par C. Ramsauer et R. Kollath d'une part et E. Brüche d'autre part (voir Fig. 3). Cette dernière, quant à elle, est proche de celle publiée par R. B. Brode [13]. Nous avons introduit tour à tour les deux sections efficaces dans notre programme. Les deux vérifient bien les mesures pour $\mathrm{CO}_{2}$ pur mais la seconde donne une meilleure concordance avec les mesures réalisées sur des mélanges $\mathrm{Ar}-\mathrm{CO}_{2}$. Aussi est-ce cette dernière que nous avons retenue. On en trouvera une paramétrisation en annexe.

R. D. Hake Jr, et A. V. Phelps [12] donnent également (Fig. 3) différentes sections efficaces vibrationnelles correspondant à des pertes d'énergie

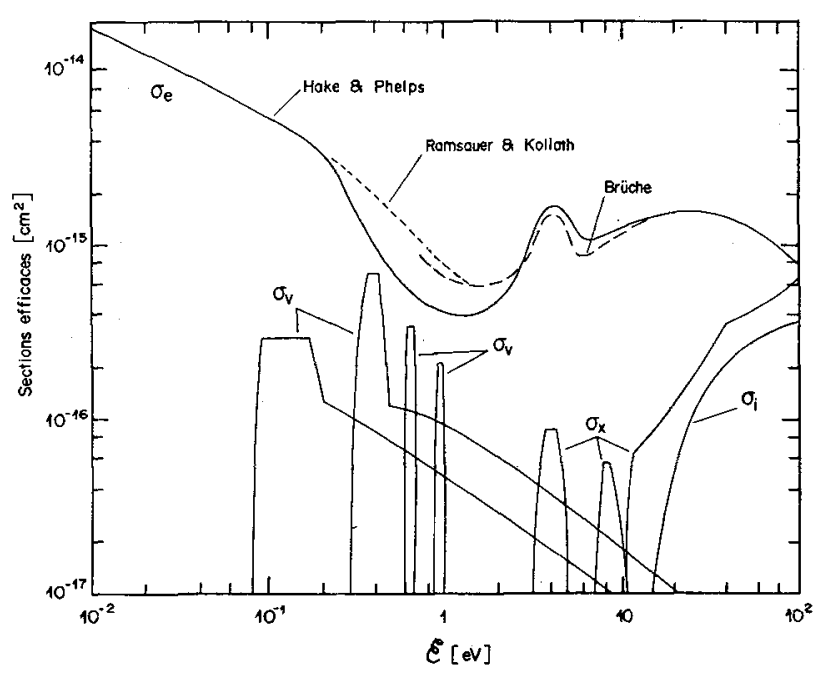

FIG. 3, - Différentes sections efficaces de collisions dans le gaz carbonique. A noter les sections efficaces de chocs vibrationnels correspondant à des pertes d'énergie de $0,08 \mathrm{eV}, 0,3 \mathrm{eV}, 0,6 \mathrm{eV}$ et $0,9 \mathrm{eV} . \sigma_{\mathrm{e}}$ et $\sigma_{x}$ et $\sigma_{\mathrm{i}}$ sont respectivement les sections efficaces de chocs élastiques, d'excitation électronique et d'ionisation.

(2) Beaucoup de mesures sur les gaz datent des années 1930. A cette époque, les différents mécanismes de collisions étaient mal connus et uniquement caractérisés par une probabilité de collisions à laquelle était associée une section efficace de collision appelée aussi section efficace totale de collision. Cette section efficace correspond pour beaucoup de gaz à la section efficace de chocs élastiques (par exemple pour l'argon) ou en est très voisine (par exemple pour le gaz carbonique, l'isobutane ou le méthane). Nous nous sommes inspirés des unes et des autres selon qu'elles sont connues. Pour notre calcul c'est formellement la section efficace de chocs élastiques $\sigma_{\mathrm{e}}$ quì nous intéresse. 
de $0,08 \mathrm{eV}, 0,3 \mathrm{eV}, 0,6 \mathrm{eV}$ et $0,9 \mathrm{eV}$. Comme le notent ces auteurs, les valeurs des sections efficaces correspondant aux raies de $0,6 \mathrm{eV}$ et $0,9 \mathrm{eV}$ doivent être fortement diminuées pour obtenir un bon accord entre la théorie et les mesures. De plus, les niveaux d'énergie rotationnelle qui apparaissent à des énergies plus faibles ne sont pas considérés. Compte tenu de ces remarques, nous avons défini un modèle simplifié de sections efficaces pour les chocs rotationnels et vibrationnels établissant une sorte de moyenne à partir de celle proposées par R. D. Hake Jr, et A. V. Phelps. Ce modèle est paramétrisé en annexe.

Sur la figure 4 nous montrons la vitesse de migration $w_{x}$ et l'énergie caractéristique $\mathscr{E}_{k}$ calculées en fonction de $E / N$. Ces courbes sont en bon accord (sauf pour $\mathscr{E}_{k}$ à $195^{\circ}$ sur environ une décade de $E / N$ ) avec les points expérimentaux relevés par diffé $\nmid$ rents auteurs et résumés dans la publication de R. D. Hake Jr, et A. V. Phelps.

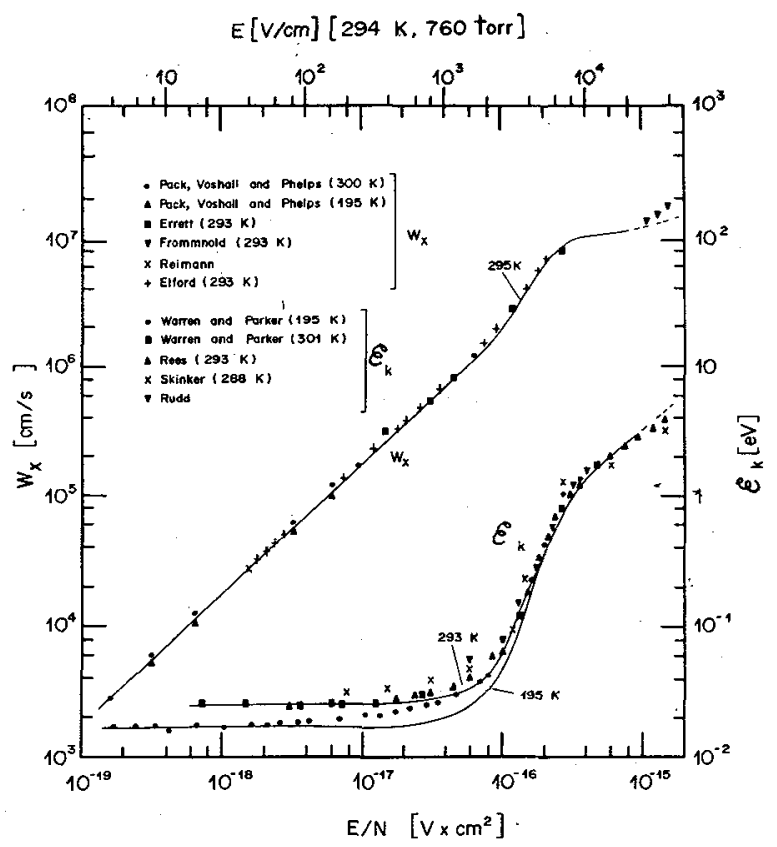

FIG. 4. - Vitesse de migration $w_{x}$ et énergie caractéristique $\mathscr{E}_{k}$ dans le gaz carbonique en fonction de $E / N$. Les courbes théoriques sont en bon accord avec les points relevés expérimentalement par différents auteurs [12]

Il faut noter que ces courbes ne sont plus valables pour $E / N>8 \times 10^{-16} \mathrm{~V} \times \mathrm{cm}^{2}$ (ce qui correspond environ à $E=20000 \mathrm{~V} / \mathrm{cm}$ à pression atmosphérique et température ambiante) à cause de l'excitation électronique (qui se produit pour des électrons d'environ $4 \mathrm{eV}$, voir figure 3) qui n'est pas envisagée dans le calcul.

Nous avons alors étudié la vitesse de migration $w_{x}$ en fonction du champ électrique $E$ pour différents mélanges $\mathrm{Ar}-\mathrm{CO}_{2}$. Seul un mélange à environ $10 \%$ de $\mathrm{CO}_{2}$ donne une vitesse constante et saturée aux champs électriques élevés (Fig. 5). Mais ce mélange est très critique et des décharges s'y produisent aisément. Aussi l'avons-nous écarté pour le fonctionnement des chambres à migration.

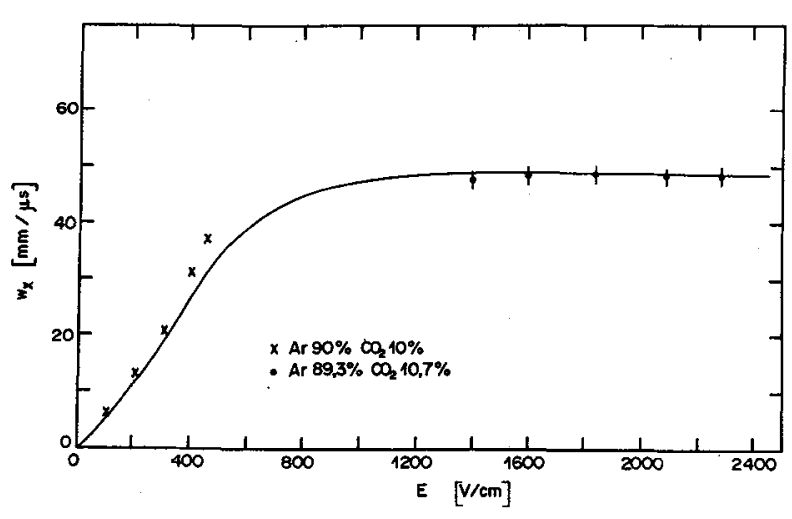

FIG. 5. - Vitesse de migration $w_{x}$ dans un mélange gazeux Ar- $\mathrm{CO}_{2}$ contenant environ $10 \%$ de $\mathrm{CO}_{2}$. La courbe a été calculée pour $10 \%$ de $\mathrm{CO}_{2}(294 \mathrm{~K}$ et $760 \mathrm{~mm}$ de $\mathrm{Hg})$. Les points expérimentaux sont tirés des références [14] $(\times)$ et [15] $(O)$.

3.3 Méthane et MélangeS Ar- $\mathrm{CH}_{4}$. - La section efficace de collisions des électrons dans le méthane n'est connue que pour des énergies supérieures à $1 \mathrm{eV}$ [16]. Nous avons été obligés d'ajuster et de compléter cette section efficace aux basses énergies à l'aide des mesures de T. E. Bortner, G. S. Hurst et W. G. Stone [17], E. B. Wagner, F. J. Davis et G. S. Hurst [18], W. N. English et G. C. Hanna [14] et J. H. Cobb [19]. Elles conduisent à une section efficace de collisions élastiques représentée à la figure 6 et paramétrisée en annexe. La section efficace de collisions rotationnelles et vibrationnelles que nous avons employée est celle qu'ont utilisée V. Palladino et B. Sadoulet [1, 2].

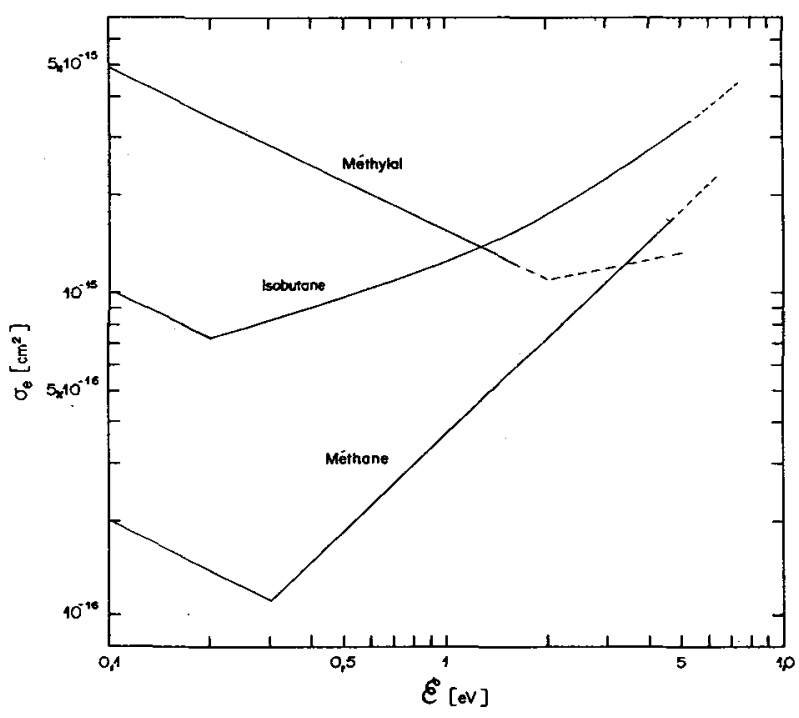

FIG. 6. - Sections efficaces de collisions élastiques dans l'isobutane, le méthane et le méthylal utilisées dans notre calcul. 
Elle est constante à basse énergie et s'annule au niveau vibrationnel le plus élevé soit $0,36 \mathrm{eV}$ (Fig. 7). Sur la figure 8, nous montrons les résultats obtenus pour le méthane pur et sur la figure 9 ceux obtenus pour un mélange de $90 \%$ Ar et $10 \%$ de $\mathrm{CH}_{4}$. Seul le méthane pur donne une vitesse de migration constante aux champs électriques élevés. Mais malheureusement ce gaz ne peut être utilisé dans une chambre à migration à cause de ces mauvaises propriétés d'amplification.

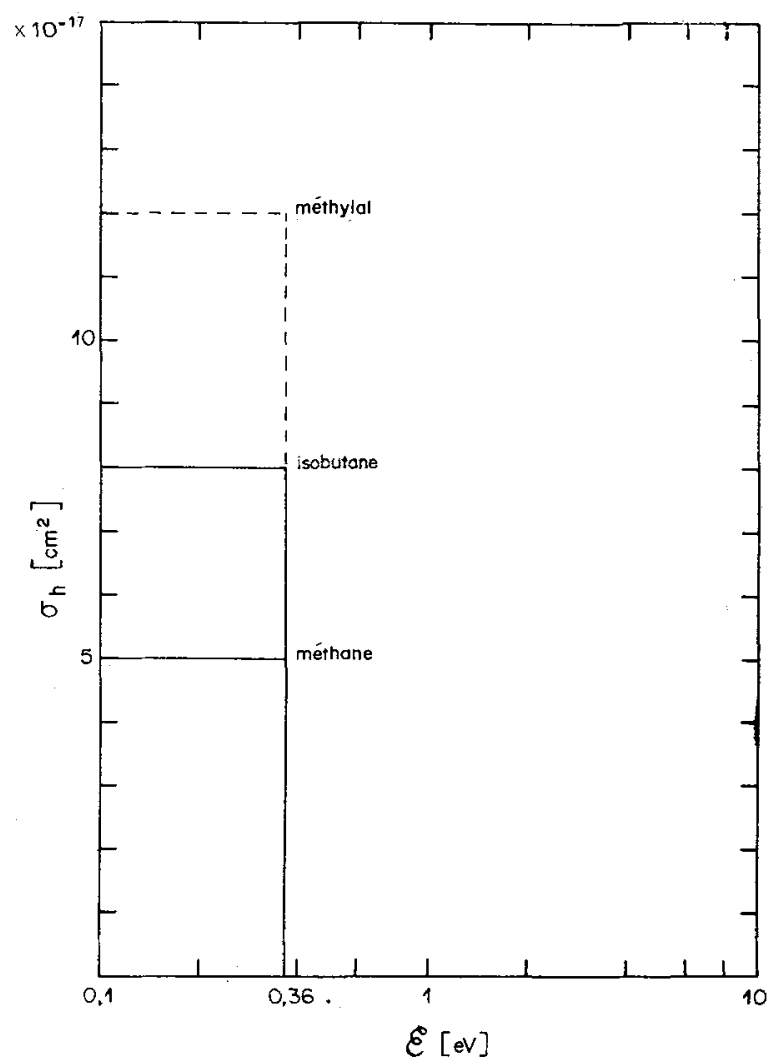

FIG. 7. - Sections efficaces de collisions rotationnelles et vibrationnelles dans l'isobutane, le méthane et le méthylal utilisés dans notre calcul.

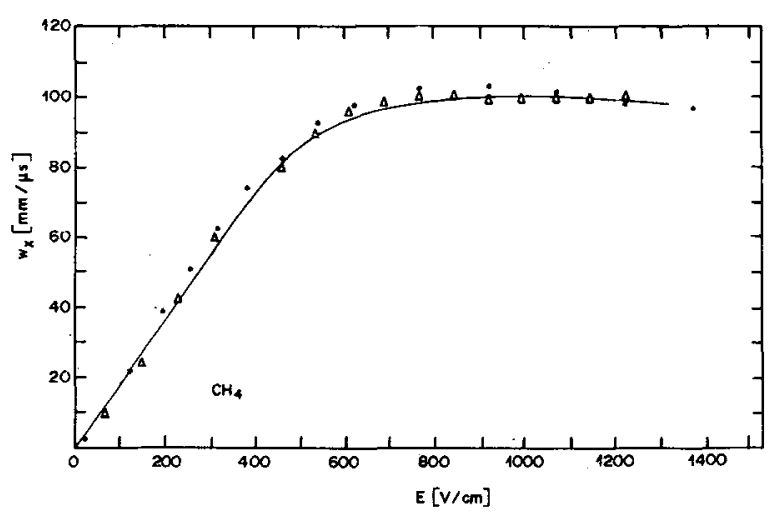

FIG. 8. - Vitesse de migration $w_{x}$ dans le méthane pur. Points expérimentaux relevés par les auteurs des références [17] $(\Delta)$ et [18] (O). (294 K et $760 \mathrm{~mm}$ de $\mathrm{Hg}$ ).

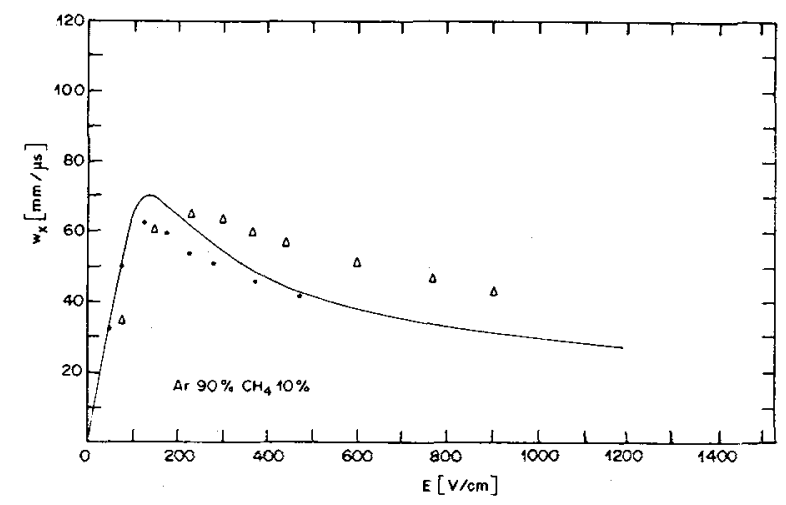

FIG. 9. - Vitesse de migration $w_{x}$ dans un mélange gazeux Ar- $\mathrm{CH}_{4}$ contenant $10 \%$ de $\mathrm{CH}_{4}$. Points expérimentaux relevés par les auteurs des références [17] $(\Delta)$ et [19] $(O)$. (294 K et $760 \mathrm{~mm}$ de $\mathrm{Hg})$.

3.4 Isobutane ET MÉLANGES Ar-IsoC $\mathrm{H}_{40}$. Les sections efficaces de l'isobutane ont été ajustées à partir de valeurs proposées par E. Brüche [16], V. Palladino et B. Sadoulet $[1,2]$ et à l'aide de mesures de vitesse de migration dans l'isobutane pur et dans différents mélanges $\mathrm{Ar}-\mathrm{IsoC}{ }_{4} \mathrm{H}_{10}$. Elles sont représentées sur les figures 6 et 7 et paramétrisées en annexe. Sur la figure 10 nous montrons les résultats obtenus pour l'isobutane pur et sur la figure 11 ceux obtenus pour un mélange de $70 \%$ d'argon et $30 \%$ d'isobutane. Contrairement à une idée bien établie, ce dernier mélange ne conduit pas à une vitesse saturée. Cependant l'adjonction d'une faible quantité de méthylal (indispensable pour éviter la dégénérescence rapide de la chambre dans le temps) $[21,22]$ améliore la saturation de la vitesse de migration des électrons.

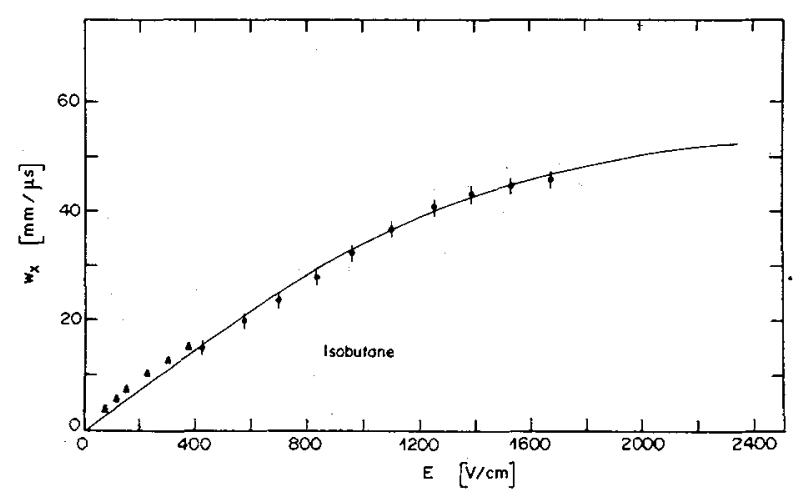

FIG. 10. - Vitesse de migration $w_{x}$ dans l'isobutane pur. Points expérimentaux relevés au C.E.R.N. [3] (๑) et par les auteurs de la référence $[20](\Delta) .(294 \mathrm{~K}$ et $760 \mathrm{~mm}$ de $\mathrm{Hg})$.

3.5 INFLUENCE DU MÉTHYLAL. - Les sections efficaces ont été ajustées à partir d'une part d'une mesure de la vitesse de migration dans le méthylal pur réalisée par A. Breskin et $\mathrm{N}$. Trautner [23] (Fig. 13), d'autre part d'une mesure que nous avons 


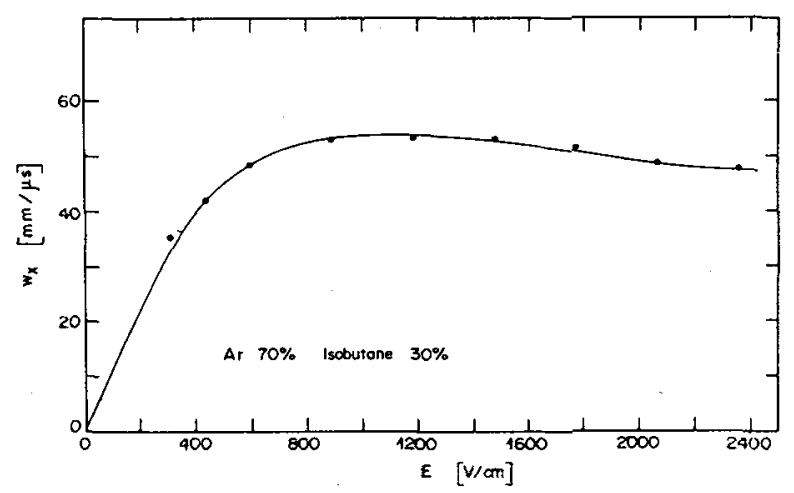

FIG. 11. - Vitesse de migration $w_{x}$ dans un mélange gazeux Ar- $\mathrm{IsoC}_{4} \mathrm{H}_{10}$ contenant $30 \%$ d'IsoC $\mathrm{C}_{4} \mathrm{H}_{10}$. Points expérimentaux relevés au C.E.R.N. [3] (294 K et $760 \mathrm{~mm}$ de $\mathrm{Hg}$ ).

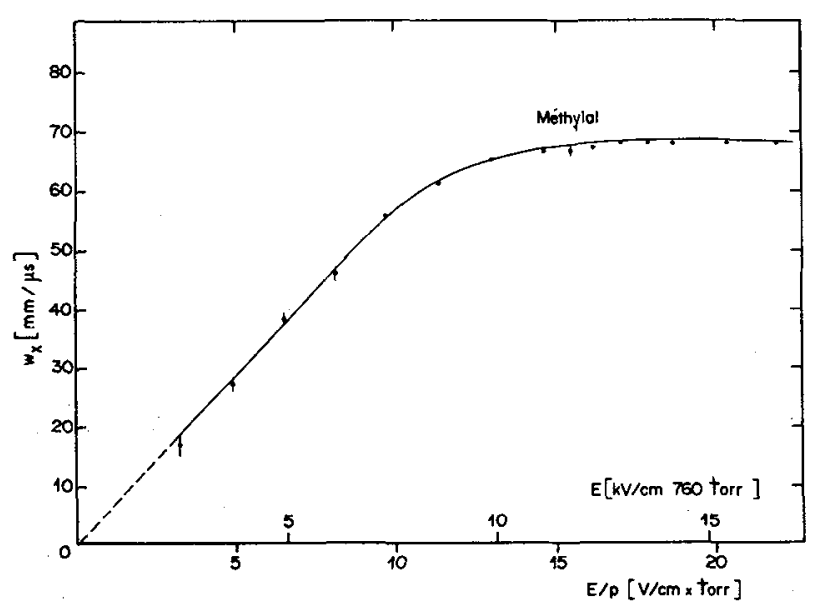

FIG. 12, - Vitesse de migration $w_{x}$ dans le méthylal pur. Courbe théorique et points expérimentaux [23] (294 K, $760 \mathrm{~mm}$ de $\mathrm{Hg})$.

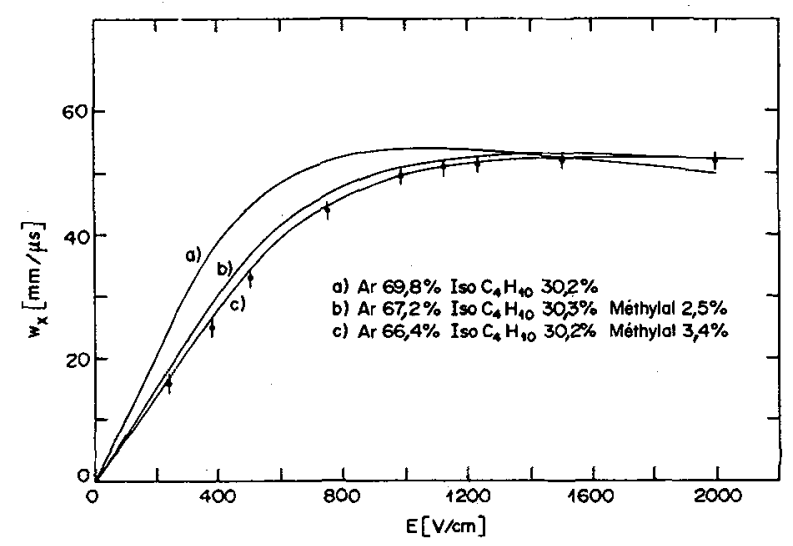

Fig. 13. - Vitesse de migration $w_{x}$ calculée pour trois mélanges d'argon, d'isobutane et de méthylal à taux d'isobutane quasi constant. Une faible quantité de méthylal fait varier fortement ces courbes aux faibles champs électriques. Les points expérimentaux sont ceux relevés pour le mélange à $3,4 \%$ de méthylal (calcul et mesures effectués à $294 \mathrm{~K}$ et $760 \mathrm{~mm}$ de $\mathrm{Hg}$ ).

effectuée avec le mélange standard de $66,4 \%$ d'argon, 30,2\% d'isobutane et 3,4\% de méthylal. La faible concentration de méthylal dans ce dernier mélange et la rareté des résultats expérimentaux nous amènent à donner les sections efficaces avec prudence (voir les figures 6 et 7 et la paramétrisation en annexe). Comme pour l'isobutane et le méthane nous avons considéré une section efficace de chocs rotationnels et vibrationnels constante s'annulant brutalement au niveau d'énergie vibrationnelle le plus élevé soit $0,36 \mathrm{eV}[24,25]$. La figure 13 montre trois courbes calculées à différents taux de méthylal. On remarque que l'apport de méthylal diminue la vitesse de migration aux faibles champs électriques. Aux champs électriques élevés, cet effet est moins important. Par contre, l'apport de méthylal rend la vitesse de migration presque constante pour $E>1350 \mathrm{~V} / \mathrm{cm}$. Cette information est très importante si on veut obtenir un fonctionnement optimal des chambres à migration et justifie notre choix pour un mélange gazeux de 66,4\% d'argon, 30,2\% d'isobutane et $3,4 \%$ de méthylal. Toutefois, le calcul prévoit une très faible décroissance de la courbe aux champs élevés, décroissance qui n'a pas été observée expérimentalement.

4. Influence des variations de pression et de température sur la vitesse de migration. - Comme nous l'avons déjà noté au début du paragraphe 3 , toute évolution de $w_{x}$ en fonction du champ électrique peut invariablement être présentée en fonction de $E / N$ ou $E / p . w_{x}$ ne varie donc pas avec $p$ si $w_{x}$ est constant quel que soit $E$.

Nous avons établi par le calcul la variation relative de la vitesse $\Delta w_{x} / w_{x}$ pour une augmentation de température de un degré à température ambiante $\left(21^{\circ} \mathrm{C}\right)$ pour le mélange standard d'une part et pour un mélange qui contient $67,2 \%$ d'argon, 30,3\% d'isobutane et $2,5 \%$ de méthylal (Fig. 14). La valeur expérimentale trouvée avec nos chambres à

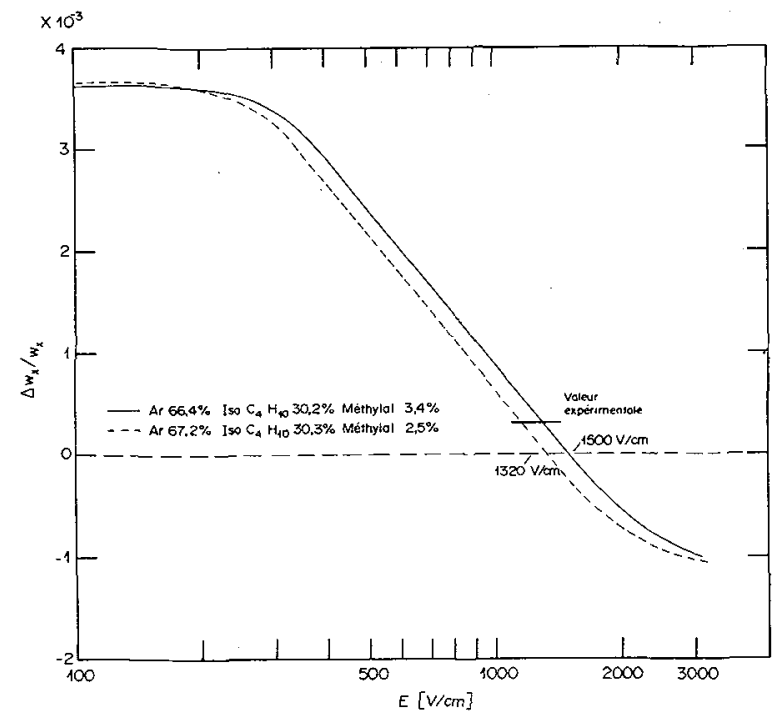

FIG. 14. - Variation de la vitesse $\Delta w_{x} / w_{x}$ dans deux mélanges gazeux pour une augmentation de température de $1^{\circ}$ à température ambiante et pression normale $(294 \mathrm{~K}$ et $760 \mathrm{~mm}$ de $\mathrm{Hg}$ ). 
migration et pour le mélange de gaz standard est de $3 \times 10^{-4}$ pour une augmentation de température de un degré à température et pression normales [5]. Ce résultat est en bon accord avec le calcul si on note que le champ électrique moyen est de $1350 \mathrm{~V} / \mathrm{cm}$ dans la grande partie de l'espace de migration. On peut noter que le passage à zéro des courbes de la figure 14 provient de la légère décroissance de $w_{x}$ aux champs électriques élevés (Fig. 13). Si cette décroissance n'existe pas ( $w_{x}$ demeure constant), la variation $\Delta w_{x} / w_{x}$ est nulle à condition que les électrons qui migrent soient suffisamment énergétiques pour qu'on puisse négliger l'agitation des molécules ou atomes de gaz dans l'établissement de la fonction de distribution des énergies ce qui est le cas avec le gaz stanđard aux champs électriques élevés.

5. Energie caractéristique et dispersion spatiale. Cette énergie est définie à la relation (9). Elle tend vers $k T$ si 1'énergie des électrons est faible et proche de l'énergie thermique des molécules de gaz. Comme nous l'avons vu à la relation (10), l'énergie caractéristique est liée à la dispersion spatiale de l'électron. Cette dispersion est souvent ramenée à un parcours de migration de un $\mathrm{cm}$; dans ce cas, on définit :

$$
\sigma_{0 x}=\sqrt{\frac{2 \mathscr{E}_{k}}{e E}}
$$

D'après la relation (10) il est clair que :

$$
\sigma_{x}=\sigma_{0 x} \sqrt{x}
$$

$\mathscr{E}_{k}$ est représentée à la figure 15 pour les cinq gaz étudiés ainsi que pour le mélange standard. On en déduit qu'il est plus intéressant d'utiliser des mélanges contenant du gaz carbonique ou du méthylal que de l'isobutane. Cependant comme nous l'avons déjà noté, le choix du gaz dans une chambre à migration est le fruit d'un compromis. Généralement, outre

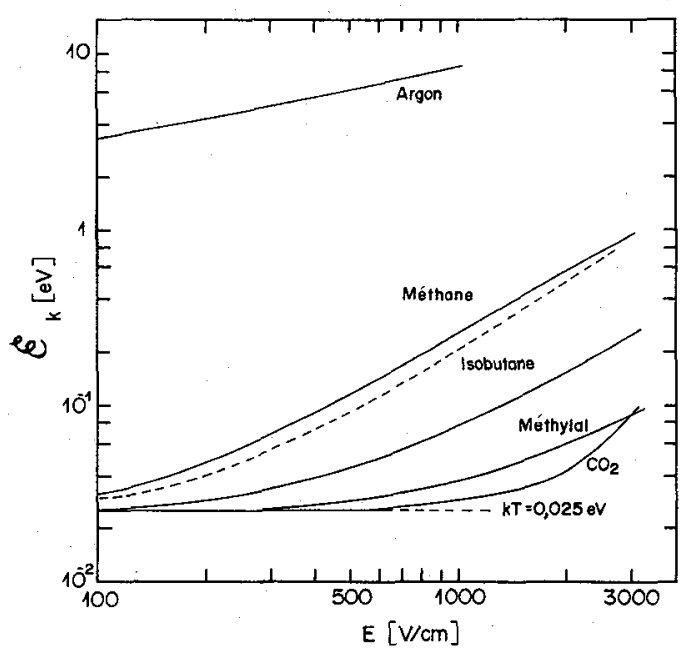

FIG. 15. - Energie caractéristique $\mathscr{E}_{k}$ pour 5 gaz purs ainsi que pour le mélange standard (en pointillés) en fonction de $E(294 \mathrm{~K}$, $760 \mathrm{~mm}$ de $\mathrm{Hg}$ ). A noter la limite thermique $\mathscr{E}_{k} \simeq 0,025 \mathrm{eV}$. une bonne précision spatiale, on recherche également une bonne stabilité de fonctionnement de la chambre, ce qui nous a fait opter pour un mélange contenant de l'isobutane (voir paragraphe 3.2).

$\sigma_{0 x}$ est représenté en fonction du champ électrique sur la figure 16 pour les cinq gaz étudiés ainsi que pour le mélange standard. Il faut noter que les relations (13) et (14) ne donnent pas la précision spatiale de détection de la chambre à migration. Celle-ci est naturellement liée à $\sigma_{x}$ mais aussi au nombre d'électrons créés dans la chambre par ionisation et au nombre d'électrons nécessaires pour déclencher les amplificateurs-discriminateurs.

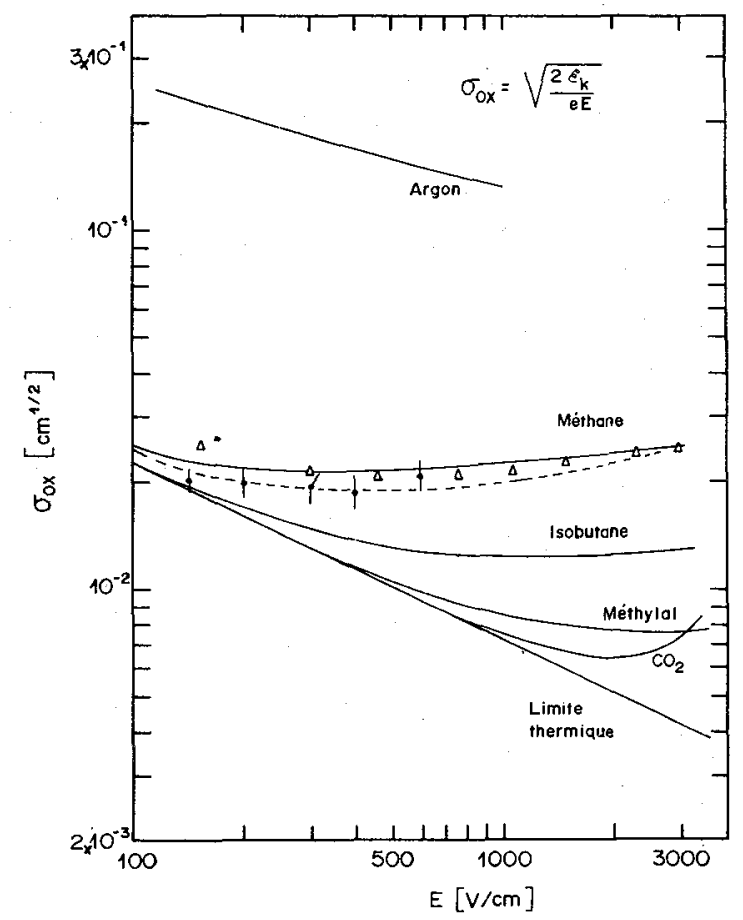

FIG. 16. - Dispersion spatiale $\sigma_{0 x}$ des électrons après $1 \mathrm{~cm}$ de migration dans 5 gaz purs ainsi que dans le mélange standard (en pointillés) en fonction de $E(294 \mathrm{~K}, 760 \mathrm{~mm}$ de $\mathrm{Hg})$. Points expérimentaux pour le méthane $(\triangle)[26]$ et pour le mélange standard (O) [27].

Les figures (17) et (18) montrent deux mesures de précision réalisées d'une part au C.E.R.N. sur un faisceau de pion de $1 \mathrm{GeV} / \mathrm{c}$ d'impulsion et à Batavia sur un faisceau de particules de $150 \mathrm{GeV} / c$ d'impulsion [28]. Les deux mesures ont été réalisées avec des chambres identiques, des bancs de test semblables et des amplificateurs-discriminateurs de performances sembiables [6].

Dans les deux cas, on peut constater que l'écart type $\sigma$ peut être la résultante de deux quantités $\sigma_{x}$ et $\sigma_{0}:$

$$
\sigma^{2}=\sigma_{x}^{2}+\sigma_{0}^{2}
$$

- $\sigma_{x}=\sqrt{2 \mathscr{E}_{k} x / e E}$ (voir relation (10)) : c'est la dispersion en position des électrons due à leur migration dans le gaz. 
- $\sigma_{0}$ est une constante liée à la dispersion due à l'électronique et à la diffusion multiple des particules.

On remarque que $\sigma_{0}$ est inférieure pour les mesures effectuées à $150 \mathrm{GeV} / c$ que pour celles effectuées à $1 \mathrm{GeV} / c$; ceci est normal car la diffusion multiple des particules diminue quand leur énergie augmente.

Les dispersions $\sigma_{x}$ déduites des deux mesures sont assez similaires et correspondent à environ $45 \mu \mathrm{m}$ pour un parcours de migration des électrons de $1 \mathrm{~cm}$.

Quelques remarques sont cependant nécessaires au sujet de cette valeur à la lumière des résultats théoriques résumés sur la figure 16. En effet, pour le gaz standard utilisé, le calcul (qui tient compte de la dispersion globale des électrons) prévoit une dispersion d'environ $200 \mu \mathrm{m}$ pour $1 \mathrm{~cm}$ de parcours. Or, pour la précision de détection d'une chambre à migration, l'important est la dispersion des tous premiers électrons qui arrivent sur l'anode et déclenchent l'amplificateur-discriminateur. Ceux-ci sont en effet suffisants pour dépasser le seuil électronique [3].

Si on considère un groupe de $n$ électrons créés ponctuellement à une distance $x$ du fil de mesure, la dispersion $\sigma_{\nu}$ du $\nu$-ième électron arrivant sur le fil de mesure est donnée par la relation approchée [29]

$$
\sigma_{\nu} \simeq \frac{\sigma_{x}}{\sqrt{2 \ln n}} \sqrt{\sum_{\nu}^{n} \frac{1}{r^{2}}}
$$

dans les conditions où $n$ est grand et $\nu \ll n$.

Compte tenu de la relation précédente et du fait que :

$$
\sum_{i}^{n+\infty} \frac{1}{r^{2}}=\frac{\pi^{2}}{6}
$$

on peut calculer la dispersion des premiers électrons arrivant sur l'anode. Pour une distance intercathode de $6 \mathrm{~mm}$ et avec le gaz standard $n$ est voisin de 72 [3] et on a par exemple $\sigma_{1}=88 \mu \mathrm{m}, \sigma_{2}=55 \mu \mathrm{m}$, $\sigma_{3}=43 \mu \mathrm{m}, \sigma_{4}=36 \mu \mathrm{m}$ pour une migration de $1 \mathrm{~cm}$.

Naturellement, ces nombres ne donnent que des ordres de grandeurs vu les hypothèses simplificatrices qui ont été faites pour établir la relation [16].

En plus, il convient de noter que les amplificateurs-discriminateurs ne déclenchent pas de façon idéale sur le $\nu$-ième électron arrivant à l'anode en un temps infiniment rapide. L'amplificateur est tributaire d'une sorte de dispersion moyenne entre les premiers électrons, qui a comme limite la dispersion du centre de gravité $\sigma_{\mathrm{G}}=\sigma_{x} / \sqrt{n}$. Pour $n=72$, on a dans notre cas $\sigma_{\mathrm{G}}=24 \mu \mathrm{m}$.

Sur les figures 17 et 18 , nous avons tracé $\sigma_{1}$ et $\sigma_{G}$ qui constituent des enveloppes pour la dispersion $\sigma_{x}$ déduites des mesures de $\sigma$ (relation 15).

Nous avons essayé d'améliorer la précision spatiale de détection en remplaçant les amplificateurs à

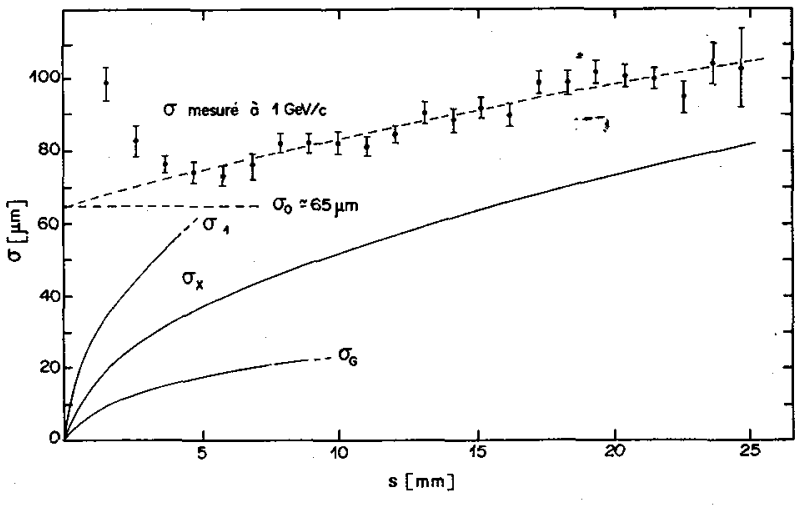

FIG. 17. - Précision spatiale $\sigma$ (écart type) mesurée avec des pions perpendiculaires à la chambre de $1 \mathrm{GeV} / c$ d'impulsion. $\sigma$ peut se décomposer en deux grandeurs $\sigma_{0}$ et $\sigma_{x}\left(\sigma^{2}=\sigma_{0}^{2}+\sigma_{x}^{2}\right)$. $\sigma_{0}$ est liée à la dispersion due à l'électronique et à la diffusion multiple des particules. $\sigma_{x}$ est liée à la migration des électrons dans la chambre. Sa valeur est comprise entre $\sigma_{1}$ (dispersion du premier électron arrivant sur l'anode) et $\sigma_{\mathrm{G}}$ (dispersion du centre de gravité des $n$ électrons arrivant sur l'anode).

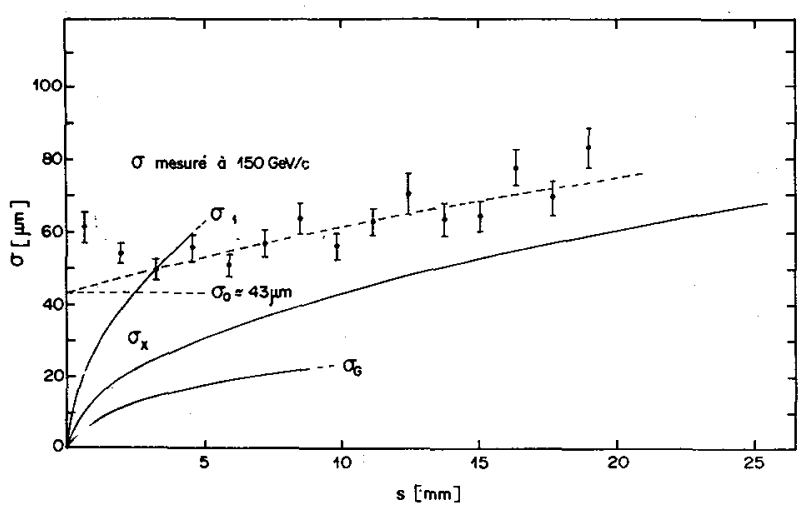

FIG. 18. - Même mesure qu'à la figure 22 mais réalisée avec des pions de $150 \mathrm{GeV} / c$ d'impulsion. $\sigma_{0}$ est inférieur à celui déterminé avec des particules de $1 \mathrm{GeV} / c$ d'impulsion.

seuil électronique fixe par des amplificateurs déclenchant sur le sommet de l'impulsion d'entrée (zéro crossing) [5]. En fait aucune différence n'a été notée; cela peut confirmer notre hypothèse que même avec un amplificateur à seuil, on obtient la dispersion moyenne des premiers électrons arrivant sur l'anode.

On remarque également sur les deux figures que $\sigma$ crô̂t au voisinage immédiat de l'anode. Ceci est dû à la dispersion statistique de la production des paires électron-ion le long de la trace ionisante.

6. Conclusions. - En vue de leur utilisation dans les chambres à migration, cinq gaz ont attiré notre attention (argon, gaz carbonique, isobutane, méthane et méthylal) ainsi que différents mélanges faisant intervenir ces gaz. Une étude du comportement des électrons dans ces gaz a été réalisée à l'aide de relations classiques simplifiées afin d'optimiser la stabilité de fonctionnement des chambres. 
Cette étude montre que le mélange 66,4\% d'argon, 30,2 \% d'isobutane et 3,4\% de méthylal que nous avons utilisé pour les chambres du C.E.R.N. [5, 6] offre un bon compromis : vitesse de migration de l'électron quasi constante pour $E>1350 \mathrm{~V} / \mathrm{cm}$ d'où faible influence de $\Delta p$ et $\Delta T$, énergie caractéristique de l'électron relativement faible d'où bonne précision spatiale de détection. En plus, ce mélange présente de bonnes propriétés d'amplification.

$\mathrm{Ce}$ travail peut être prolongé ultérieurement à d'autres gaz intéressants pour les chambres à migration (notamment à l'éthylène et à l'éthane) et le calcul peut être étendu au cas où l'électron drift est soumis à une force de Lorentz due à une induction magnétique $B$.

\section{ANNEXE}

Paramétrisation des sections efficaces de collisions intervenant dans le calcul de la vitesse de migration des électrons

1. ARGON.

$$
\begin{array}{rlrl}
\mathscr{E} & <0,3 \mathrm{eV} & \sigma_{\mathrm{e}}=1,46 \times 10^{-17}+1,24 \times 10^{-12}(0,3-\mathscr{E})^{6,5} \mathrm{~cm}^{2} \\
0,3<\mathscr{E}<1,15 \mathrm{eV} & \sigma_{\mathrm{e}}=1,46 \times 10^{-17}+1,9 \times 10^{-16}(\mathscr{E}-0,3)^{2} \mathrm{~cm}^{2} \\
1,15<\mathscr{E}<11,5 \mathrm{eV} & \sigma_{\mathrm{e}}=1,52 \times 10^{-15} \mathscr{E} / 11,5 \mathrm{~cm}^{2} \\
\mathscr{E}>11,5 \mathrm{eV} & \sigma_{\mathrm{e}}=1,52 \times 10^{-15} \times(\mathscr{E} / 11,5)^{-1 / 2} \mathrm{~cm}^{2}
\end{array}
$$

Comme souligné dans lè texte, les chocs à excitation électronique ne sont pas pris en compte dans le calcul.

2. GAZ CARBONIQUE.

$$
\begin{array}{cl}
\mathscr{E}<0,2 \mathrm{eV} & \sigma_{\mathrm{e}}=1,70 \times 10^{-15} \mathscr{E}-0,5 \mathrm{~cm}^{2} \\
0,2<\mathscr{E}<1,32 \mathrm{eV} & \sigma_{\mathrm{e}}=7,60 \times 10^{-16} \mathscr{E}-1 \mathrm{~cm}^{2} \\
1,32<\mathscr{E}<3,25 \mathrm{eV} & \sigma_{\mathrm{e}}=1,00 \times 10^{-15}-3,66 \times 10^{-16}(3,25-\mathscr{E})^{0,222} \mathrm{~cm}^{2} \\
3,25<\mathscr{E}<4,2 \mathrm{eV} & \sigma_{\mathrm{e}}=1,53 \times 10^{-15}-5,90 \times 10^{-16}(4,2-\mathscr{E})^{2,09} \mathrm{~cm}^{2} \\
4,2<\mathscr{E}<6 \mathrm{eV} & \sigma_{\mathrm{e}}=1,53 \times 10^{-15}-4,84 \times 10^{-16}(\mathscr{E}-4,2)^{0,528} \mathrm{~cm}^{2} \\
6<\mathscr{E}<25 \mathrm{eV} & \sigma_{\mathrm{e}}=1,60 \times 10^{-15}-1,75 \times 10^{-18}(25-\mathscr{E})^{2,05} \mathrm{~cm}^{2} \\
\mathscr{E}>25 \mathrm{eV} & \sigma_{\mathrm{e}}=1,60 \times 10^{-15}-2,94 \times 10^{-18}(\mathscr{E}-25)^{1,30} \mathrm{~cm}^{2}
\end{array}
$$

Comme souligné dans le texte, un modèle simple a été retenu pour $\sigma_{h}$ :

$$
\begin{array}{ll}
\mathscr{E} \geq 0,2 \mathrm{eV} & \sigma_{h}=0,35 \times 10^{-16} \mathscr{E}-0,25 \mathrm{~cm}^{2} \\
\mathscr{E}<0,2 \mathrm{eV} & \sigma_{h}=4 \times 10^{-16} \mathrm{~cm}^{2}
\end{array}
$$

3. MÉthane.

$$
\begin{aligned}
\mathscr{E} & <0,3 \mathrm{eV} & \sigma_{\mathrm{e}} & =6,25 \times 10^{-7} \times \mathscr{E}^{-1 / 2} \mathrm{~cm}^{2} \\
0,3<\mathscr{E} & <8 \mathrm{eV} & \sigma_{\mathrm{e}} & =3,69 \times 10^{-16} \times \mathscr{E}, 975 \mathrm{~cm}^{2} \\
\mathscr{E} & >8 \mathrm{eV} & & \sigma_{\mathrm{e}}=2,80 \times 10^{-15}(\mathscr{E} / 8)^{-1 / 2} \mathrm{~cm}^{2} \\
\mathscr{E} & \leq 0,36 \mathrm{eV} & \sigma_{h} & =5 \times 10^{-17} \mathrm{~cm}^{2}
\end{aligned}
$$

4. ISOBUTANE.

$$
\begin{aligned}
\mathscr{E}<0,2 \mathrm{eV} & \sigma_{e}=0,72 \times 10^{-15}(0,20 / \mathscr{E})^{1 / 2} \mathrm{~cm}^{2} \\
0,2<\mathscr{E}<0,6 \mathrm{eV} & \sigma_{e}=0,72 \times 10^{-15} \times(\mathscr{E} / 0,2)^{0,335} \mathrm{~cm}^{2} \\
0,6<\mathscr{E}<8 \mathrm{eV} & \sigma_{e}=\left[1,04 \times 10^{-15}(8-\mathscr{E})+4,8 \times 10^{-15}(\mathscr{E}-0,6)\right] / 7,4 \mathrm{~cm}^{2} \\
\mathscr{E}>8 \mathrm{eV} & \sigma_{e}=4,8 \times 10^{-15}(\mathscr{E} / 8)^{-1 / 2} \mathrm{~cm}^{2} \\
\mathscr{E} \leq 0,36 \mathrm{eV} & \sigma_{h}=8 \times 10^{-17} \mathrm{~cm}^{2}
\end{aligned}
$$




\section{MÉTHYLAL.}

$$
\begin{aligned}
\mathscr{E}<2 \mathrm{eV} & \sigma_{\mathrm{e}}=1,1 \times 10^{-15}(2 / \mathscr{E})^{1 / 2} \mathrm{~cm}^{2} \\
2<\mathscr{E}<4 \mathrm{eV} & \sigma_{\mathrm{e}}=\left[1,1 \times 10^{-15}(10-\mathscr{E})+1,8 \times 10^{-15}(\mathscr{E}-2)\right] / 8 \mathrm{~cm}^{2} \\
\mathscr{E}<4 \mathrm{eV} & \sigma_{\mathrm{e}}=1,275 \times 10^{-15}(\mathscr{E} / 4)^{0,220} \mathrm{~cm}^{2} \\
\mathscr{E} \leq 0,36 \mathrm{eV} & \sigma_{h}=12 \times 10^{-17} \mathrm{~cm}^{2}
\end{aligned}
$$

\section{Bibliographie}

[1] Palladino, V. et SAdouler, B., Lawrence Berkeley Laboratory Report (1974) LBL-3013.

[2] Palladino, V. et Sadoulet, B., Nucl. Instrum. Methods 128 (1975) 323.

[3] Schultz, G., Thèse soutenue à l'Université Louis Pasteur de Strasbourg le 8.7.1976 (No d'ordre: 1015).

[4] GRESSER, J. et SCHULTZ, G., A study of transport coefficients of electrons in some gases used in proportional and drift chambers Soumis à Nucl. Instrum. Methods.

[5] Breskin, A., Charpak, G., Gabioud, B., Sauli, F., TrautNER, N., DUINker, W. et SchUltz, G., Nucl. Instrum. Methods 119 (1974) 9.

[6] Breskin, A., Charpak, G., Sauli, F., Atkinson, M. et SCHUlTZ, G., Nucl. Instrum. Methods 124 (1975) 189.

[7] Morse, P. M., Allis, W. P. et Lamar, E. S., Phys, Rev. 48 (1935) 412 .

[8] Frost, L. S. et Phelps, A. V., Phys. Rev. 127 (1962) 1621.

[9] Parker, J. H. et Lowke, J. J., Phys. Rev. 181 (1969) 290 et 302.

[10] Engherardt, A. G. et Phelps, A. V., Phys. Rev. 133A (1964) 375 .

[11] PaCk, J. L., Voshall, R. E. et Phelps, A. V., Tables of drift velocities of slow electrons, Westinghouse Research Laboratories Report 62-928-113-R1 (1961).

[12] Hake, R. D., Jr. et Phelps, A. V., Phys. Rev. 158 (1967) 70.

[13] Brode, R. B., Rev. Mod. Phys. 5 (1933) 257.

[14] English, W. N. et Hanna, G. C., Can. J. Phys. 31 (1953) 768.

[15] Ezban, R. et MORganti, M., Internal Report C.E.R.N. NP/OM 666 (1974).

[16] Bríche, E., Ann. Phys. 4 (1930) 387.
[17] Bortner, T. E., Hurst, G. S. et Stone, W. G., Rev. Sci, Instrum. 28 (1957) 103.

[18] Wagner, E. B., Davis, F. J. et Hurst, G. S., J. Chem. Phys. 47 (1967) 3138.

[19] CoBb, J. H., ISIS Internal note 15, Nuclear Phys. Lab, Oxford (1974).

[20] Christophorou, L. G., Hurst, G. S. et Hadjiantoniou, A., J. Chem. Phys. 449 (1966) 3506.

[21] Charpak, G., Fischer, G., Minten, A., NaumanN, L., Sauli, F., Flugge, CotTfried, Ch. et Tirler, R., Nucl. Instrum. Methods 97 (1971) 377.

[22] Charpak, G., Fischer, G., Gruhn, G. R., Minten, A. Sauli, F., Plch, G. et Flugge, G., Nucl. Instrum. Methods 99 (1972) 279.

[23] Breskin, A. et Trautner, N., Nucl. Instrum. Methods 134 (1976) 35.

[24] Wladislaw, B., Grora, A. et Vicentini, G., J. Chem. Soc. (B), (1966) 586.

[25] Le Brumant, J., Marsault, J. P. et Freymann, R., C.R. Hebd. Séan. Acad. Sci., C, 263 (1966) 833.

[26] Cochran, L. W. et Forester, D. W., Phys. Rev. 126 (1962) 1785.

[27] Breskin, A., Charpak, G. et Sauli, F., Nucl. Instrum. Methods 136 (1976) 497.

[28] Nigmanov, T. S., Pugachevich, V. P., Riabtsov, V. D., Shafranov, M. D., TSYGanov, E. N., URALSKY, D. V., VODOPIANOV, A. S., SAULI, F., ATAC, M. et TOMPKINS, J., Fermilab Internal Report 76/26 EX1.

[29] Cramer, H., Mathematical Methods of Statistics (Princeton University Press) 1966 , p. 374. 